\title{
ACÚMULO DE METAIS PESADOS EM CANA-DE-AÇÚCAR MEDIANTE A APLICAÇÃO DE LODO DE ESGOTO E VINHAÇA
}

\section{FÁBIO CAMILOTTI ${ }^{1}$, MARCOS O. MARQUES ${ }^{2}$, ITAMAR ANDRIOLI ${ }^{3}$, ALYSSON R. DA SILVA ${ }^{1}$, LUIZ C. TASSO JUNIOR ${ }^{4}$, FABIO O. DE NOBILE}

\begin{abstract}
RESUMO: O uso de resíduos agroindustriais pode acumular metais pesados no solo e na planta. O objetivo deste trabalho foi avaliar os efeitos do lodo de esgoto e/ou vinhaça, aplicados no solo, disponibilizando $\mathrm{Cd}, \mathrm{Cr}$, $\mathrm{Ni}$ e $\mathrm{Pb}$ ao solo e quantificar as respectivas concentrações em plantas de cana-de-açúcar, após três aplicações anuais sucessivas. O experimento foi conduzido em condições de campo, com parcelas experimentais de cinco linhas espaçadas de $1,5 \mathrm{~m}$ e $10 \mathrm{~m}$ de comprimento. O delineamento experimental adotado foi o de blocos casualizados, com três repetições. Os tratamentos avaliados foram: 1 . Lodo de esgoto com $100 \%$ de N; 2. Lodo de esgoto com $200 \%$ de $\mathrm{N} ; 3$. Vinhaça com $100 \%$ de K; 4. Vinhaça com $200 \%$ de K; 5 . Lodo de esgoto+vinhaça com $100 \%$ de $\mathrm{N}$ e K; 6. Lodo de esgoto+vinhaça com $200 \%$ de $\mathrm{N}$ e K; 7 . Testemunha (fertilização mineral). Os baixos teores de metais pesados $(\mathrm{Cd}, \mathrm{Cr}, \mathrm{Ni}$ e $\mathrm{Pb})$ disponíveis no solo e nas partes das plantas de cana-de-açúcar indicam que o lodo de esgoto e a vinhaça, empregados nas doses mencionadas, não apresentaram, após três aplicações anuais sucessivas, potencial de contaminação do sistema soloplanta.
\end{abstract}

PALAVRAS-CHAVE: cádmio, chumbo, cromo.

\section{HEAVY METALS ACCUMULATION IN SUGARCANE AFTER APPLICATION IN SEWAGE SLUDGE AND VINASSE}

\begin{abstract}
The organic residues are able to change heavy metals in soil and plant. The objective of this work was to evaluate the sewage sludge and/or vinasse effects in $\mathrm{Cd}, \mathrm{Cr}, \mathrm{Ni}$ and $\mathrm{Pb}$ soil valuable contents and sugarcane accumulated amounts after three successive annual applications. The experiment was conducted in field conditions. The experimental plots were made of five plant lines spaced of $1.5 \mathrm{~m}$, with $10 \mathrm{~m}$ of length. The experimental design was a randomized blocks with three replications. The treatments were: 1. Sewage sludge (100\% of N); 2. Sewage sludge $(200 \%$ of $N)$; 3. Vinasse $(100 \%$ of K); 4. Vinasse $(200 \%$ of K); 5. Sewage sludge plus vinasse (100\% of $\mathrm{N}$ and $\mathrm{K}$ ); 6 . Sewage sludge plus vinasse (200\% of N and K); 7 . Control (mineral fertilization to sugarcane plants). The small soil heavy metal contents and small sugarcane heavy metal accumulated amounts it can be draw that the used residues, at these doses, do not present, until this moment, soil-plant system contamination potential.
\end{abstract}

KEYWORDS: cadmium, lead, nickel.

\section{INTRODUÇÃO}

O termo metal pesado pode ser atribuído aos elementos químicos que apresentam massa específica superior a $5 \mathrm{~g} \mathrm{~cm}^{-3}$ (MELO et al., 1997). Entre esses, encontram-se mais de 20 elementos que, em relação aos seres vivos, podem ou não ter importância. Desse modo, os nutrientes $\mathrm{Cu}, \mathrm{Fe}$ e $\mathrm{Zn}$ também são classificados como metais pesados. Elementos como cádmio e

\footnotetext{
${ }^{1}$ Eng ${ }^{\circ}$ Agrônomo, Doutorando em Agronomia, Departamento de Solos e Adubos, UNESP, Jaboticabal - SP, camilotti@bol.com.br, Bolsista da CAPES.

${ }^{2}$ Prof. Adjunto, Departamento de Tecnologia, UNESP, Jaboticabal - SP.

${ }^{3}$ Prof. Adjunto, Departamento de Solos e Adubos, UNESP, Jaboticabal - SP.

${ }^{4}$ Eng $^{\mathrm{O}}$ Agrônomo, Doutorando em Agronomia, Departamento de Tecnologia, UNESP, Jaboticabal - SP. Bolsista da CAPES.

${ }^{5}$ Eng ${ }^{\mathrm{o}}$ Agrônomo, Doutorando em Agronomia, Departamento de Engenharia Rural, UNESP, Jaboticabal - SP.

Recebido pelo Conselho Editorial em: 30-1-2006
}

Aprovado pelo Conselho Editorial em: 19-3-2007 
chumbo, até o momento, não demonstraram qualquer relação de importância; níquel e cromo, de outra forma, podem trazer benefícios em determinadas circunstâncias (MARQUES et al., 2002).

$\mathrm{Na}$ natureza, os metais pesados podem originar-se das rochas. Os solos são resultantes de processos de intemperismo de rochas e, dessa forma, acabam por conter os metais pesados herdados da rocha-matriz (FASSBENDER, 1980). Entretanto, nos solos cultivados, os fertilizantes minerais também podem ser possíveis fontes de metais pesados (FASSBENDER, 1980 e SIMONETE, 2001). Além disso, o uso de resíduos orgânicos, como fertilizantes e/ou condicionadores do solo, também pode contribuir para a adição de metais pesados nos solos (MARQUES et al., 1998; MARQUES et al, 2002; GATTO, 2003). LAKE (1987) relaciona 28 elementos metálicos pesados, intervalos de níveis de ocorrência em solos agrícolas e valores de maior freqüência. Segundo esse autor, o cádmio ocorre nos solos em teores totais variando de 0,01 a $0,70 \mathrm{mg} \mathrm{kg}^{-1}$; o cromo de 5 a $1.000 \mathrm{mg} \mathrm{kg}$; o níquel de 5 a $500 \mathrm{mg} \mathrm{kg}^{-1}$ e o chumbo de 2 a $200 \mathrm{mg} \mathrm{kg}^{-1}$.

Para que um determinado elemento presente no solo possa ser absorvido pelas plantas, há necessidade de o mesmo se encontrar em forma disponível, ou seja, solúvel na solução do solo. Dessa forma, a presença de matéria orgânica, de óxidos e de hidróxidos de alumínio, ferro e manganês, ou seja, o tipo e a composição do solo, as características e as propriedades das substâncias orgânicas e das inorgânicas presentes, o valor e as variações do $\mathrm{pH}$, o potencial redox do solo e a especiação química do metal em questão são fatores que interferem na disponibilidade do elemento (MATTIAZZO-PREZOTTO, 1994 e MARQUES et al., 2002). Além disso, a presença da planta e os fenômenos de troca que se verificam na rizosfera também devem ser considerados.

De acordo com LAKE (1987), quando o teor de um determinado metal num resíduo é menor ou igual ao teor do mesmo metal no solo, pode-se inferir que o resíduo não apresenta potencial de contaminação. Entretanto, o autor ressalta que, quando se trata de resíduos orgânicos, a mineralização da matéria orgânica, que poderá ocorrer após a disposição no solo, tenderá a aumentar os riscos de contaminação.

No que se refere à potencialidade de resíduos atuar como fonte de metais pesados, RIBEIRO FILHO et al. (2001), trabalhando com Latossolo Vermelho-Amarelo distrófico utilizado para a disposição de rejeito de uma companhia de extração e industrialização de zinco, encontraram teores de $156 \mathrm{mg} \mathrm{kg}^{-1}$ de $\mathrm{Cd}$ e $551 \mathrm{mg} \mathrm{kg}^{-1}$ de $\mathrm{Pb}$ obtidos pela soma de cinco extrações de fracionamento seqüencial. SILVA et al. (2002), estudando alternativas agronômicas para o lodo de esgoto do Distrito Federal, não encontraram contaminação de $\mathrm{Cd}$ e $\mathrm{Pb}$ com aplicações de $54 \mathrm{t} \mathrm{ha}^{-1}$ de lodo úmido. Aumento nos teores de Ni (extração em DTPA) na camada de 0-10 cm em um Latossolo Vermelho Eutroférrico foram obtidos por GALDOS et al. (2004), após duas aplicações de lodo de esgoto.

Em algumas situações em que os metais se encontram disponíveis no solo, a absorção e a translocação do elemento por meio dos tecidos das plantas podem ocorrer de forma reduzida ou mesmo não ocorrer. Isso se explica por meio de mecanismos de proteção das plantas, os quais bloqueiam o elemento quando esse ainda se encontra no solo (complexos formados com os exsudatos das raízes) ou mesmo quando o elemento já se encontra no interior das raízes onde são barrados por camadas de células que compõem a endoderme (MARQUES et al., 2002).

Apesar das possibilidades para a indisponibilidade dos metais no solo e dos mecanismos de proteção das plantas, a absorção de metais pesados, dependendo das condições, pode ocorrer em quantidades suficientes para o surgimento de sintomas de fitotoxicidade. LAKE (1987) e MELO et al. (1997) estabeleceram os seguintes níveis fitotóxicos para $\mathrm{Cd}$ (2-10 $\left.\mathrm{mg} \mathrm{kg}^{-1}\right), \mathrm{Cr}$ (10$\left.100 \mathrm{mg} \mathrm{kg}^{-1}\right)$, Ni (11-100 $\left.\mathrm{mg} \mathrm{kg}^{-1}\right)$ e Pb (35-400 $\left.\mathrm{mg} \mathrm{kg}^{-1}\right)$.

Considerando a presença de elementos nutrientes e metais pesados na composição do lodo de esgoto, ao mesmo tempo em que o seu emprego como fertilizante e/ou condicionador de solo pode trazer vantagens por promover a reciclagem de nutrientes e melhoria da fertilidade do solo, pode 
também trazer prejuízos se, por ventura, viabilizar aumento na absorção e acúmulo de metais pesados em plantas (SILVA et al., 1998).

O presente trabalho teve por objetivo avaliar os teores dos metais pesados $\mathrm{Cd}, \mathrm{Cr}, \mathrm{Ni}$ e $\mathrm{Pb}$ no solo e em diferentes partes de plantas de cana-de-açúcar cultivadas com lodo de esgoto e vinhaça, aplicados anualmente durante um período de três anos.

\section{MATERIAL E MÉTODOS}

O estudo foi desenvolvido em área da Destilaria Santa Inês Ltda., município de Pontal - SP. O solo da área foi classificado como Latossolo Vermelho distroférrico argiloso, cujas características para fins de fertilidade estão apresentadas na Tabela 1 . O clima da região é do tipo Cwa pela classificação de Köeppen.

TABELA 1. Caracterização química e granulométrica do solo $(0-20 \mathrm{~cm})$.

\begin{tabular}{|c|c|c|c|c|c|c|c|c|c|c|c|c|}
\hline $\begin{array}{c}\mathrm{pH} \\
\mathrm{CaCl}_{2}\end{array}$ & MO & $\begin{array}{c}\mathrm{P} \\
\text { resina }\end{array}$ & $\mathrm{K}$ & $\mathrm{Ca}$ & $\mathrm{Mg}$ & $\mathrm{H}+\mathrm{Al}$ & SB & तTC & $\mathrm{V}$ & $\Delta$ reir & Silte & Argila \\
\hline & $\mathrm{g} \mathrm{dm}$ & $\mathrm{mg} \mathrm{dm}^{-3}$ & & & & $1 \mathrm{dm}$ & & & $\%$ & & $\mathrm{~g} \mathrm{~kg}^{-1}$ & \\
\hline 5,0 & 25 & 20 & 1,2 & 20 & 6 & 28 & 27,2 & 55,2 & 49 & 380 & 110 & 510 \\
\hline
\end{tabular}

No mês de abril de 2000, foi feito o plantio da cana-de-açúcar (variedade SP 81-3250), cuja área vinha sendo cultivada com cana-de-açúcar e milho para fins de ensilagem na reforma do canavial.

O experimento constou de sete tratamentos, com três repetições, distribuídas em delineamento em blocos ao acaso. A área total de cada unidade experimental (parcela) foi de $75 \mathrm{~m}^{2}$ (cinco linhas de cana-de-açúcar, cada uma com comprimento de $10 \mathrm{~m}$, e espaçamento entre linhas de $1,5 \mathrm{~m})$.

A quantificação da concentração de metais pesados no lodo de esgoto utilizado em cada ciclo de cana-de-açúcar está descrita na Tabela 2.

TABELA 2. Teores de metais pesados no lodo de esgoto utilizados em cada ciclo da cana-deaçúcar.

\begin{tabular}{|c|c|c|c|c|}
\hline \multirow{2}{*}{ Ciclo da Cultura } & $\mathrm{Cd}$ & $\mathrm{Cr}$ & $\mathrm{Ni}$ & $\mathrm{Pb}$ \\
\hline & \multicolumn{4}{|c|}{$\mathrm{mg} \mathrm{kg}^{-1}$} \\
\hline $1^{\mathrm{o}}$ corte & 1,54 & 41,33 & 34,75 & 43,84 \\
\hline $2^{\underline{o}}$ corte & 1,54 & 41,33 & 34,75 & 43,84 \\
\hline $3^{\underline{o}}$ corte & 1,50 & 41,00 & 34,33 & 42,42 \\
\hline
\end{tabular}

Os tratamentos consistiram de duas doses de lodo de esgoto e de duas doses de vinhaça, sendo os resíduos aplicados separadamente ou em conjunto em área total (Tabela 3).

O lodo de esgoto foi aplicado anualmente para fornecer 100 ou $200 \%$ de todo $\mathrm{N}$ requerido pela de cana-de-açúcar, e a vinhaça foi aplicada anualmente para fornecer 100 ou $200 \%$ de todo K requerido pela de cana-de-açúcar, conforme recomendação de SPIRONELLO et al. (1997). Vários são os fatores que interferem na percentagem de $\mathrm{N}$ disponível para as plantas presente no lodo de esgoto: sistema de tratamento a que foi submetido o esgoto, sistema de estabilização do lodo gerado, percentagem de $\mathrm{N}-\mathrm{NO}_{3}^{-}$e $\mathrm{N}^{-\mathrm{NH}_{4}^{+}}$e método de aplicação no solo (FRANKLIN, 2004). $\mathrm{Na}$ maior parte dos casos, esse valor oscila entre 30 e $35 \%$, mas pode chegar até $50 \%$ em casos isolados. Neste trabalho, considerou-se de $33 \%$ a percentagem de $\mathrm{N}$ disponível às plantas presentes no lodo empregado. O lodo de esgoto foi obtido na Estação de Tratamento de Esgoto da Sabesp de Franca - SP, e a vinhaça, na Destilaria Santa Inês. 
TABELA 3. Doses aplicadas de lodo de esgoto e vinhaça a cada ciclo da cana-de-açúcar em função dos tratamentos.

\begin{tabular}{|c|c|c|c|c|}
\hline \multirow{2}{*}{ Tratamento } & \multicolumn{3}{|c|}{ Ciclo da Cana-de-Açúcar } & \multirow{2}{*}{ Total } \\
\hline & $1^{\mathrm{o}}$ corte & $2^{-}$corte & $3^{-}$corte & \\
\hline Le $1^{(1)}, \mathrm{tha}^{-1}$ & 5 & 7 & 7,5 & 19,5 \\
\hline Le $2^{(2)}, \mathrm{t} \mathrm{ha}^{-1}$ & 10 & 14 & 15 & 39 \\
\hline $\mathrm{V} 1^{(3)}, \mathrm{m}^{3} \mathrm{ha}^{-1}$ & 83 & 86 & 95 & 264 \\
\hline $\mathrm{V} 2^{(4)}, \mathrm{m}^{3} \mathrm{ha}^{-1}$ & 166 & 172 & 190 & 528 \\
\hline Le $1+\mathrm{V} 1$ & $5+83$ & $7+86$ & $7,5+95$ & $19,5+264$ \\
\hline Le $2+\mathrm{V} 2$ & $10+166$ & $14+338$ & $15+190$ & $39+528$ \\
\hline Testemunha $^{(5)}$ & - & - & - & - \\
\hline
\end{tabular}

Para a vinhaça os teores de metais pesados estiveram abaixo do limite de determinação do método analítico empregado, motivo pelo qual não foram apresentados. Nos tratamentos em que o lodo de esgoto foi fonte de $\mathrm{N}$, o $\mathrm{K}$ foi suplementado na forma de $\mathrm{KCl}$, enquanto, quando se usou vinhaça como fonte de $\mathrm{K}$, o $\mathrm{N}$ foi suplementado na forma de uréia. Nos tratamentos com os dois resíduos, uréia e $\mathrm{KCl}$ não foram empregados. $\mathrm{Na}$ testemunha, todo $\mathrm{N}$ e $\mathrm{K}$ requeridos pela cana-deaçúcar foram fornecidos com uréia e $\mathrm{KCl}$, respectivamente. Os resíduos orgânicos foram aplicados em área total após cada colheita da cultura. Em seguida, as soqueiras foram cultivadas com cultivador de uma haste com ponteiras aletadas. A cultura foi mantida no limpo por meio de aplicações de herbicida (hexazinona + diuron).

As amostras de solo foram coletadas nas entrelinhas, nas camadas de $0-10 ; 10-20 ; 20-30 ; 30$ 40 e 40-50 cm de profundidade, após o terceiro corte da cana-de-açúcar, secas ao ar, peneiradas (malha de $2 \mathrm{~mm}$ ) e submetidas à digestão com $\mathrm{HNO}_{3}+\mathrm{H}_{2} \mathrm{O}_{2}+\mathrm{HCl}$ (USEPA, 1986). Nos extratos obtidos, procederam as leituras para $\mathrm{Cd}, \mathrm{Cr}, \mathrm{Ni}$ e $\mathrm{Pb}$ por meio de espectrofotometria de absorção atômica.

As plantas de cana-de-açúcar foram divididas em colmos, folhas e palmitos. Os colmos foram desintegrados em desintegrador tipo forrageiro e as folhas e os palmitos foram lavados. As diferentes partes foram mantidas em estufa para secagem até peso constante, moídos em moinho tipo Willey, pesadas e submetidas à digestão nítrico-perclórica, de acordo com a metodologia de BATAGLIA et al. (1983). Nos extratos, foram determinados $\mathrm{Cd}$, $\mathrm{Cr}$, $\mathrm{Ni}$ e $\mathrm{Pb}$ por espectrofotometria de absorção atômica. Os resultados obtidos foram submetidos à análise de variância.

\section{RESULTADOS E DISCUSSÃO}

Na Tabela 4, estão apresentados os resumos da análise de variância para os dados obtidos nas diferentes camadas do solo.

Os teores de $\mathrm{Cd}$ e $\mathrm{Pb}$ nas amostras do solo, comparados com os valores considerados normais para solos (LAKE, 1987), ressaltam o potencial desses lodos em contaminar o solo com cádmio, uma vez que os teores apresentados, expressos com base na matéria seca do lodo, estão acima dos limites considerados normais para solos. Contudo, essa expectativa não se confirmou, pois, embora os teores de cádmio nos solos tenham ocorrido em níveis elevados, não se pôde inferir que se trata de contaminação por parte dos tratamentos testados, uma vez que o tratamento-testemunha apresentou teores na mesma ordem de grandeza dos demais.

Os teores de $\mathrm{Cd}$ e $\mathrm{Pb}$ encontrados nos solos após o terceiro corte demonstram que o solo se apresentava com teores de cádmio acima da faixa considerada normal. LAKE (1987) destaca que os solos, em geral, apresentam teores de Cd variando no intervalo entre 0,01 e $0,70 \mathrm{mg} \mathrm{kg}^{-1}$ e teores 
de $\mathrm{Pb}$ entre 2 e $200 \mathrm{mg} \mathrm{kg}^{-1}$. RIBEIRO FILHO et al. (2001), analisando amostras de Latossolo Vermelho-Amarelo distrófico, utilizado para disposição de rejeitos do processo de extração e industrialização de zinco, obtiveram teores de $\mathrm{Cd}$ e $\mathrm{Pb}$ muito superiores aos encontrados nesta pesquisa. De forma contrária e concordante com os resultados aqui obtidos, SILVA et al. (2002) não encontraram contaminações de $\mathrm{Cd}$ e $\mathrm{Pb}$ em solo que recebeu lodo de esgoto.

TABELA 4. Resumo da análise de variância (ANOVA) dos teores de $\mathrm{Cd}, \mathrm{Cr}, \mathrm{Ni}$ e $\mathrm{Pb}$ em diferentes camadas do solo.

\begin{tabular}{|c|c|c|c|c|}
\hline ANOVA & $\mathrm{Cd}$ & $\mathrm{Cr}$ & $\mathrm{Ni}$ & $\mathrm{Pb}$ \\
\hline & \multicolumn{4}{|c|}{$0-10 \mathrm{~cm}$} \\
\hline & $1,60 \mathrm{NS}^{(1)}$ & $2,83 \mathrm{NS}$ & $0,82 \mathrm{NS}$ & $0,55 \mathrm{NS}$ \\
\hline \multirow[t]{2}{*}{ C.V. $(\%)^{(2)}$} & 7 & 8 & 9 & 5 \\
\hline & \multicolumn{4}{|c|}{$10-20 \mathrm{~cm}$} \\
\hline Teste F & $2,29 \mathrm{NS}$ & $1,67 \mathrm{NS}$ & $0,52 \mathrm{NS}$ & $0,39 \mathrm{NS}$ \\
\hline \multirow[t]{2}{*}{ C.V. (\%) } & 6 & 11 & 10 & 6 \\
\hline & \multicolumn{4}{|c|}{$20-30 \mathrm{~cm}$} \\
\hline Teste F & $2,50 \mathrm{NS}$ & $1,74 \mathrm{NS}$ & $0,57 \mathrm{NS}$ & $1,28 \mathrm{NS}$ \\
\hline \multirow[t]{2}{*}{ C.V. (\%) } & 7 & 11 & 8 & 4 \\
\hline & \multicolumn{4}{|c|}{$30-40 \mathrm{~cm}$} \\
\hline Teste F & $1,33 \mathrm{NS}$ & $0,20 \mathrm{NS}$ & $1,08 \mathrm{NS}$ & $0,59 \mathrm{NS}$ \\
\hline \multirow[t]{2}{*}{ C.V. (\%) } & 10 & 14 & 9 & 6 \\
\hline & \multicolumn{4}{|c|}{$40-50 \mathrm{~cm}$} \\
\hline Teste F & $1,36 \mathrm{NS}$ & $0,05 \mathrm{NS}$ & $1,82 \mathrm{NS}$ & $0,56 \mathrm{NS}$ \\
\hline C.V. (\%) & 11 & 15 & 8 & 6 \\
\hline
\end{tabular}

Os teores de cromo encontrados nos solos agrícolas variam de 5 a $1.000 \mathrm{mg} \mathrm{kg}^{-1}$, sendo $100 \mathrm{mg} \mathrm{kg}^{-1}$ o valor mais freqüente (LAKE, 1987). Os resultados encontrados neste trabalho, em todas as camadas analisadas, situam-se entre $26,08 \mathrm{e} 39,75 \mathrm{mg} \mathrm{kg}^{-1}$. SILVA et al. (1998) obtiveram resultados inferiores a esses quando se utilizaram de lodo de esgoto na cultura da cana-de-açúcar. OLIVEIRA \& MATTIAZZO (2001) constataram, na camada de 0-20 cm de um Latossolo Amarelo textura média sem aplicação de lodo de esgoto, teores de 15,55 a $16,57 \mathrm{mg} \mathrm{kg}^{-1}$, inferiores ao do presente estudo.

$\mathrm{Na}$ Tabela 5, são relacionados os teores médios disponíveis dos metais pesados analisados nas amostras de solo, nas diferentes profundidades coletadas, após a realização do terceiro corte da cana-de-açúcar.

Os lodos empregados no presente trabalho apresentaram em suas composições teores de níquel próximos a $35 \mathrm{mg} \mathrm{kg}$, permitindo a inferência acerca da sua baixa potencialidade de contaminação dos solos. Corroborando essas expectativas, os teores de níquel encontrados no solo (13 a $16 \mathrm{mg} \mathrm{kg}^{-1}$ ) podem ser considerados normais para solos cultivados. Com base nos teores encontrados no tratamento-testemunha e nos resultados de ROVERS et al. (1983), os quais destacam o teor de $40 \mathrm{mg} \mathrm{kg}^{-1}$, como média para Latossolos do Estado de São Paulo, estes autores verificaram que o uso de resíduos não proporcionou aumento dos níveis de níquel no solo acima dos valores comumente encontrados. GALDOS et al. (2004), após duas aplicações anuais de lodo de esgoto em Latossolo Vermelho eutroférrico, nas doses de 21,6 e 20,5 t ha ${ }^{-1}$, respectivamente, encontraram teores máximos de $0,9 \mathrm{mg} \mathrm{dm}^{-3}$, após a segunda aplicação. Esses teores são inferiores aos encontrados na pesquisa em questão.

No que se refere à comparação dos tratamentos, nas camadas de solo amostradas, observa-se que não houve efeito dos tratamentos sobre a disponibilidade dos metais pesados nos solos, após a realização do terceiro corte. MARQUES (1996), empregando lodo de esgoto proveniente da ETE Suzano/SABESP, na dose de 41,6 $\mathrm{t} \mathrm{ha}^{-1}$, em Latossolo Vermelho-Escuro, textura média, cultivado 
com cana-de-açúcar, encontrou os seguintes teores de metais pesados no solo: $\mathrm{Cr}=23,06$; $\mathrm{Ni}=$ $2,74 \mathrm{e} \mathrm{Pb}=10,04$, expressos em $\mathrm{mg} \mathrm{dm}^{-3}$.

TABELA 5. Teores totais de $\mathrm{Cd}, \mathrm{Cr}$, $\mathrm{Ni}$ e $\mathrm{Pb}$ em diferentes camadas do solo após o terceiro corte de cana-de-açúcar em função dos tratamentos.

\begin{tabular}{|c|c|c|c|c|}
\hline Tratamento & $\mathrm{Cd}$ & $\mathrm{Cr}$ & $\mathrm{Ni}$ & $\mathrm{Pb}$ \\
\hline & \multicolumn{4}{|c|}{$\begin{array}{l}-\mathrm{mg} \mathrm{kg}^{-1}- \\
0-10 \mathrm{~cm}\end{array}$} \\
\hline Le $1^{(1)}$ & 1,8 & 27,1 & 14,6 & 32,3 \\
\hline $\operatorname{Le} 2^{(2)}$ & 1,7 & 33,4 & 16,2 & 32,5 \\
\hline $\mathrm{V} 1^{(3)}$ & 1,8 & 29,9 & 14,8 & 31,5 \\
\hline $\mathrm{V} 2^{(4)}$ & 1,8 & 29,2 & 14,1 & 32,2 \\
\hline Le $1^{(1)}+\mathrm{V} 1^{(3)}$ & 1,8 & 31,9 & 15,2 & 32,9 \\
\hline Le $2^{(2)}+\mathrm{V} 2^{(4)}$ & 1,9 & 27,7 & 14,4 & 32,1 \\
\hline \multirow[t]{2}{*}{ Testemunha $^{(5)}$} & 1,9 & 29,7 & 14,9 & 31,0 \\
\hline & \multicolumn{4}{|c|}{$10-20 \mathrm{~cm}$} \\
\hline Le 1 & 1,8 & 29,7 & 14,8 & 32,8 \\
\hline Le 2 & 1,7 & 29,3 & 16,0 & 30,9 \\
\hline V 1 & 1,8 & 29,0 & 14,8 & 32,7 \\
\hline V 2 & 1,9 & 29,0 & 14,2 & 32,6 \\
\hline Le $1+\mathrm{V} 1$ & 1,8 & 28,3 & 15,6 & 32,9 \\
\hline Le $2+\mathrm{V} 2$ & 1,8 & 30,0 & 15,3 & 32,2 \\
\hline \multirow[t]{2}{*}{ Testemunha } & 1,9 & 29,7 & 15,6 & 31,9 \\
\hline & \multicolumn{4}{|c|}{$20-30 \mathrm{~cm}$} \\
\hline Le 1 & 2,0 & 28,1 & 15,7 & 32,9 \\
\hline Le 2 & 1,8 & 34,1 & 15,8 & 31,4 \\
\hline V 1 & 2,0 & 30,4 & 15,2 & 31,8 \\
\hline V 2 & 1,7 & 27,4 & 14,9 & 32,2 \\
\hline Le $1+\mathrm{V} 1$ & 1,8 & 29,7 & 16,3 & 32,1 \\
\hline Le $2+$ V 2 & 1,8 & 33,4 & 14,8 & 32,2 \\
\hline \multirow[t]{2}{*}{ Testemunha } & 1,8 & 31,8 & 15,8 & 30,2 \\
\hline & \multicolumn{4}{|c|}{$30-40 \mathrm{~cm}$} \\
\hline Le 1 & 1,9 & 29,8 & 15,3 & 32,1 \\
\hline Le 2 & 1,7 & 37,5 & 15,3 & 31,6 \\
\hline V 1 & 1,8 & 30,0 & 15,0 & 31,5 \\
\hline V 2 & 1,8 & 30,8 & 15,1 & 32,8 \\
\hline Le $1+\mathrm{V} 1$ & 1,8 & 32,9 & 16,2 & 32,0 \\
\hline Le $2+\mathrm{V} 2$ & 1,9 & 29,8 & 13,8 & 33,0 \\
\hline \multirow[t]{2}{*}{ Testemunha } & 2,0 & 33,1 & 16,0 & 30,4 \\
\hline & \multicolumn{4}{|c|}{$40-50 \mathrm{~cm}$} \\
\hline Le 1 & 1,8 & 30,2 & 14,7 & 31,7 \\
\hline Le 2 & 1,5 & 30,0 & 14,7 & 30,7 \\
\hline V 1 & 1,6 & 29,4 & 14,0 & 31,0 \\
\hline V 2 & 1,6 & 29,9 & 14,0 & 32,3 \\
\hline Le $1+\mathrm{V} 1$ & 1,6 & 32,8 & 15,3 & 31,3 \\
\hline Le $2+$ V 2 & 1,8 & 30,7 & 13,0 & 32,3 \\
\hline Testemunha & 1,8 & 30,6 & 15,7 & 30,0 \\
\hline
\end{tabular}

A comparação dos teores médios dos diferentes metais, nas partes das plantas, em função dos tratamentos testados, permite inferir que os valores encontrados não sofreram influência dos tratamentos (Tabela 6). 
$\mathrm{Na}$ Tabela 7, encontram-se os teores de metais em partes das plantas de cana-de-açúcar. $\mathrm{O}$ cromo foi detectado no colmo e no palmito, e o níquel, por sua vez, no palmito e nas folhas, demonstrando que a translocação do cromo no interior das plantas é mais difícil do que a do níquel. O chumbo é o único, dentre os metais analisados, que foi detectado em todas as partes avaliadas da cana-de-açúcar. Não foram detectados os metais $\mathrm{Cd}$, nas diferentes partes da plantas, $\mathrm{Cr}$, na folha, e $\mathrm{Ni}$, no colmo, por estarem abaixo do limite de detecção pelo método de extração empregado.

TABELA 6. Resumo da análise de variância (ANOVA) dos teores totais de $\mathrm{Cd}, \mathrm{Cr}, \mathrm{Ni}$ e $\mathrm{Pb}$ em partes da cana-de-açúcar no terceiro corte.

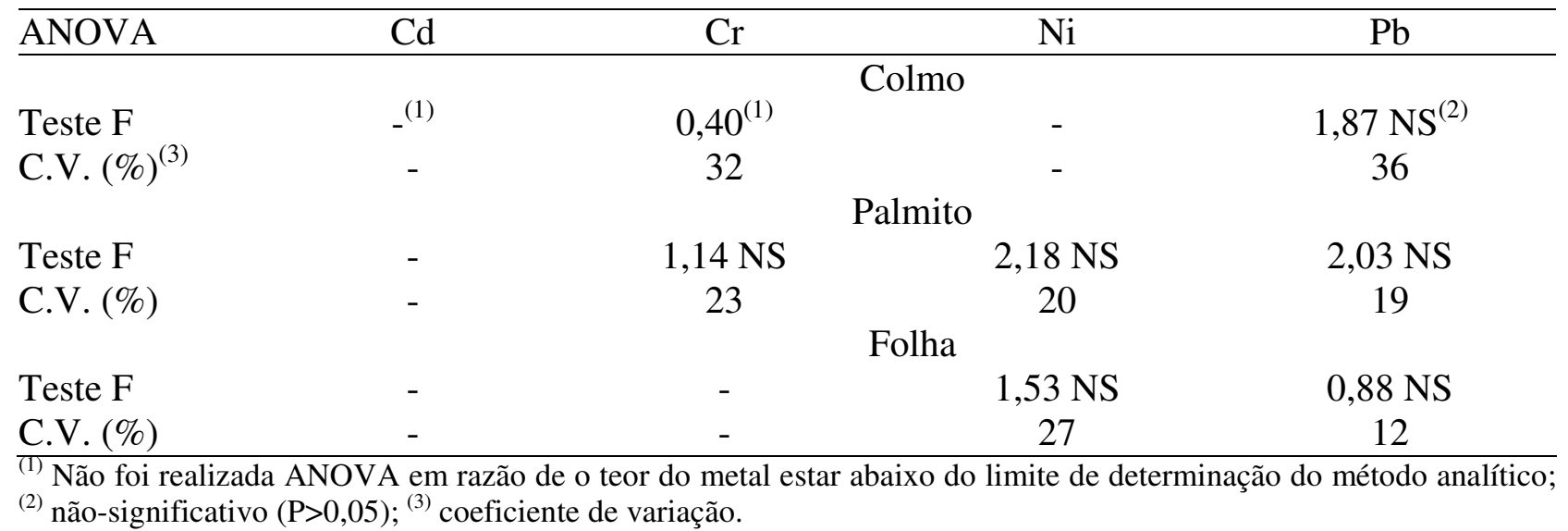

TABELA 7. Efeito de tratamentos nos valores médios de $\mathrm{Cd}, \mathrm{Cr}$, $\mathrm{Ni}$ e $\mathrm{Pb}$ em partes da cana-deaçúcar, na avaliação após as colheitas do terceiro corte.

\begin{tabular}{|c|c|c|c|c|}
\hline \multirow{2}{*}{ Tratamento } & $\mathrm{Cd}$ & $\mathrm{Cr}$ & $\mathrm{Ni}$ & $\mathrm{Pb}$ \\
\hline & \multicolumn{4}{|c|}{$\mathrm{mg} \mathrm{k}^{-1}$} \\
\hline & \multicolumn{4}{|c|}{ Colmo } \\
\hline Le $1^{(1)}$ & $-(6)$ & 2,0 & - & 1,3 \\
\hline Le $2^{(2)}$ & - & 1,7 & - & 2,3 \\
\hline $\mathrm{V} 1^{(3)}$ & - & 1,7 & - & 1,3 \\
\hline $\mathrm{V} 2^{(4)}$ & - & 1,3 & - & 1,7 \\
\hline Le $1^{(1)}+\mathrm{V} 1^{(3)}$ & - & 1,7 & - & 2,3 \\
\hline Le $2^{(2)}+\mathrm{V} 2^{(4)}$ & - & 1,7 & - & 1,3 \\
\hline \multirow[t]{2}{*}{ Testemunha $^{(5)}$} & - & 1,7 & - & 1,3 \\
\hline & \multicolumn{4}{|c|}{ Palmito } \\
\hline Le 1 & - & 1,7 & 4,0 & 14,0 \\
\hline Le 2 & - & 1,7 & 4,7 & 15,0 \\
\hline V 1 & - & 2,0 & 2,7 & 10,3 \\
\hline V 2 & - & 2,0 & 4,0 & 17,0 \\
\hline Le $1+\mathrm{V} 1$ & - & 1,3 & 4,3 & 13,7 \\
\hline Le $2+$ V 2 & - & 1,7 & 3,7 & 16,3 \\
\hline \multirow[t]{2}{*}{ Testemunha } & - & 2,0 & 3,3 & 16,0 \\
\hline & \multicolumn{4}{|c|}{ Folha } \\
\hline Le 1 & - & - & 2,3 & 19,3 \\
\hline Le 2 & - & - & 2,3 & 17,0 \\
\hline V 1 & - & - & 1,7 & 20,7 \\
\hline V 2 & - & - & 1,7 & 18,7 \\
\hline Le $1+$ V 1 & - & - & 2,0 & 20,0 \\
\hline Le $2+$ V 2 & - & - & 1,3 & 20,3 \\
\hline Testemunha & - & - & 2,0 & 19,3 \\
\hline
\end{tabular}


Deve-se ressaltar que os níveis de metais encontrados neste trabalho, nas plantas de cana-deaçúcar, estão aquém dos valores mínimos necessários para o surgimento de sintomas de toxicidade em plantas (LAKE, 1987 e MELO et al., 1997). A baixa ocorrência ou mesmo a ausência de metais nas partes aéreas de plantas de cana-de-açúcar também foi relatada por SILVA et al. (2000), os quais, analisando plantas cultivadas em solos que receberam, no ano anterior, lodo de esgoto nas doses de 20; 40 e $80 \mathrm{t} \mathrm{ha}^{-1}$, não detectaram a presença de metais nas partes aéreas das plantas, porém verificaram o acúmulo desses elementos nas raízes. Comportamentos dessa natureza podem ser explicados pelos mecanismos diversos de proteção das plantas aos metais pesados (MARQUES et al., 2002).

Observa-se, na Tabela 8, que as quantidades aplicadas de $\mathrm{Cd}, \mathrm{Cr}$, $\mathrm{Ni}$ e $\mathrm{Pb}$ na área foram baixas em comparação ao máximo permitido. Para serem atingidos os valores acumulados máximos de $\mathrm{Cd}$, Cr, Ni e $\mathrm{Pb}$, seriam necessários, respectivamente, mais 3.900; 10.843; 1.880 e 1.071 aplicações anuais de lodo de esgoto em dose suficiente para fornecer todo o $\mathrm{N}$ exigido pela cana-de-açúcar.

TABELA 8. Metais pesados adicionados ao solo até o terceiro corte da cana-de-açúcar pela aplicação cumulativa de 25,5 e $51 \mathrm{t} \mathrm{ha}^{-1}$ de lodo de esgoto (Le) e quantidade acumulada máxima permitida desses elementos, em solos agrícolas, devido à adição do resíduo.

\begin{tabular}{|c|c|c|c|}
\hline \multirow{2}{*}{ Metal Pesado } & \multicolumn{2}{|c|}{ Quantidade Aplicada do Metal } & \multirow{2}{*}{$\begin{array}{l}\text { Quantidade Acumulada } \\
\text { Máxima Permitida }\end{array}$} \\
\hline & $19,5 \mathrm{t} \mathrm{ha}^{-1} \mathrm{de} \mathrm{Le}^{(1)}$ & $39 \mathrm{t} \mathrm{ha}^{-1} \mathrm{de} \mathrm{Le}^{(2)}$ & \\
\hline & & & $\mathrm{kg} \mathrm{ha}^{-1}$ \\
\hline $\mathrm{Cd}$ & 0,03 & 0,06 & $39^{(3)}$ \\
\hline $\mathrm{Cr}$ & 0,83 & 1,66 & $3.000^{(4)}$ \\
\hline $\mathrm{Ni}$ & 0,67 & 1,34 & $420^{(3)}$ \\
\hline $\mathrm{Pb}$ & 0,84 & 1,68 & $300^{(3)}$ \\
\hline
\end{tabular}

${ }^{\text {(1) }}$ dose cumulativa de aplicações anuais de lodo de esgoto para fornecer $100 \%$ do $\mathrm{N}$ requerido pela cultura; ${ }^{(2)}$ dose cumulativa de aplicações anuais de lodo de esgoto para fornecer $200 \%$ do $\mathrm{N}$ requerido pela cultura; ${ }^{(3)}$ CETESB (1999); ${ }^{(4)}$ CFR (1993).

\section{CONCLUSÕES}

Os baixos teores de metais pesados $(\mathrm{Cd}, \mathrm{Cr}, \mathrm{Ni}$ e $\mathrm{Pb})$ disponíveis no solo e nas partes das plantas de cana-de-açúcar indicam que o lodo de esgoto e a vinhaça empregados nas doses mencionadas não apresentaram, após três aplicações anuais sucessivas, potencial de contaminação do sistema solo-planta.

\section{REFERÊNCIAS}

BATAGLIA, O.C.; FURLANI, A.M.C.; TEIXEIRA, J.P.F.; FURLANI, P.R.; GALLO, J.R. Métodos de análise química de plantas. Campinas: Instituto Agronômico, 1983. 48 p. (Boletim Técnico, 78)

CETESB. COMPANHIA DE TECNOLOGIA DE SANEAMENTO AMBIENTAL. Aplicação de lodos de sistemas de tratamento biológico em áreas agrícolas - critérios para projeto e operação. São Paulo, 1999. 32 p. (Manual Técnico da Norma P4230).

CFR. CODE OF FEDERAL REGULATIONS. Title 40, chapter I, Subchapter O - Sewage Sludge. Part 503. Standards for the use or disposal of sewage sludge. 1993. p.9.387-415, (Federal Register, 48).

FASSBENDER, H.W. Química de suelos com énfasis em suelos de América Latina. San José: Matilde de la Cruz, 1980. 398 p.

FRANKLIN, R. Land application of sewage sludge. Clemson: Faculty of Soils and Land Resources, Clemson University, 2004. 16 p. 
GALDOS, M.V.; MARIA, I.C.; CAMARGO, O.A. Atributos químicos e produção de milho em um Latossolo Vermelho eutroférrico tratado com lodo de esgoto. Revista Brasileira de Ciência do Solo, Viçosa, v.28, n.3, p.569-77, 2004.

GATTO, R.H. Lodo de esgoto e vinhaça como fonte de cálcio, magnésio e potássio para a cultura da cana-de-açúcar. 2003. 107 f. Dissertação (Mestrado em Produção Vegetal) - Faculdade de Ciências Agrárias e Veterinárias, Universidade Estadual Paulista, Jaboticabal, 2003.

LAKE, D.L. Sludge disposal to land. In: LESTER, J.N. Heavy metals in wastewater and sludge treatment process. Boca Raton: CRC Press, 1987. v.2, p.91-130.

MARQUES, M.O. Incorporação de lodo de es goto em solo cultivado com cana-de-açúcar. 1996. 111 f. Tese (Livre-Docência) - Faculdade de Ciências Agrárias e Veterinárias, Universidade Estadual Paulista, Jaboticabal, 1996.

MARQUES, M.O.; MELO, W.J.; BELLINGIERI, P.A.; MARRETO, G.H.; KANESIRO, M.A.B.; MARQUES, T.A.; CHELLI, R.A.; LEITE, S.A.S. Heavy metals in soil and sugarcane as affected by sewage sludge. In: WORLD CONGRESS OF SOIL SCIENCE, 16., 1998, Montpellier. Anais... Montpellier: Association Internatioanale de al Science du Sol, 1998. 1 CD-ROM.

MARQUES, M.O.; MELO, W.J.; MARQUES, T.A. Metais pesados e o uso de biossólido na agricultura. In: TSUTIYA, M.T.; COMPARINI, J.B.; ALÉM SOBRINHO, P.; HESPANHOL, I.; CARVALHO, P.C.T.; MELFI, A.J.; MELO, W.J.; MARQUES, M.O. Biossólidos na agricultura. 2.ed. São Paulo: ABES/SP, 2002. p.365-403.

MATTIAZZO-PREZOTTO, M.E. Comportamento do cobre, cádmio, crômio, níquel e zinco adicionados a solos de clima tropical em diferentes valores de pH. 1994. $197 \mathrm{f}$. Tese (LivreDocência) - Escola Superior de Agricultura "Luiz de Queiroz", Universidade de São Paulo, Piracicaba, 1994.

MELO, W.J.; MARQUES, M.O.; SILVA, F.C.; BOARETTO. A.E. Uso de resíduos sólidos urbanos na agricultura e impactos ambientais. In: CONGRESSO BRASILEIRO DE CIÊNCIA DO SOLO, 26., 1997, Rio de Janeiro. Anais.... Campinas: Sociedade Brasileira de Ciência do Solo, 1997.1 CD-ROM

OLIVEIRA, F.C.; MATTIAZZO, M.E. Metais pesados em Latossolo tratado com lodo de esgoto e em plantas de cana-de-açúcar. Scientia Agricola, Piracicaba, v.51, n.3, p.581-93, 2001.

RIBEIRO FILHO, M.R.; SIQUEIRA, J.O.; CURI, N.; SIMÃO, J.B.P. Fracionamento e biodisponibilidade de metais pesados em solo contaminado, incubado com materiais orgânicos e inorgânicos. Revista Brasileira de Ciência do Solo, Viçosa, v.25, n.2, p. 495-507, 2001.

ROVERS, H.; CAMARGO, O.A.; VALADARES, J.M.A.S. Níquel total e solúvel em DTPA em solos do Estado de São Paulo. Revista Brasileira de Ciência do Solo, Campinas, v.2, n.3, p. 217-20, 1983.

SILVA, F.C.; BOARETTO, A.E.; BERTON, R.S.; ZOTELLI, H.B.; PEXE, C.A.; MENDONÇA, E. Cana-de-açúcar cultivada em solo adubado com lodo de esgoto: nutrientes, metais pesados e produtividade. Pesquisa Agropecuária Brasileira, Brasília, v.33, n.1, p.1-8, 1998.

SILVA, F.C.; FANTE JUNIOR, L.; PILOTTO, J.E.; RODRIGUES, J.A.; BOARETTO, A.E.; OLIVEIRA, J.C.M.; BERTON, R.S.; ZOTELLI, H.B. Evaluating the residual effects of sludge in root distribution an heavy metals in sugar cane crop. International Sugar Journal, Glamorgan, v.102, n.1220, p.424-30, 2000.

SILVA, J.E.; RESCK, D.V.S.; SHARMA, R.D. Alternativa agronômica para o biossólido produzido no Distrito Federal. I - Efeito na produção de milho e na adição de metais pesados em Latossolo no cerrado. Revista Brasileira de Ciência do Solo, Viçosa, v.26, n.2, p.487-95, 2002.

SIMONETE, A.A. Alterações nas propriedades químicas de um Argissolo adubado com lodo de esgoto e desenvolvimento e acúmulo de nutrientes em plantas de milho. 2001. $89 \mathrm{f}$. Tese 
(Doutorado em Solos e Nutrição Vegetal) - Escola Superior de Agricultura "Luiz de Queiroz", Universidade de São Paulo, Piracicaba, 2001.

SPIRONELLO, A.; RAIJ, B. Van; PENATTI, C.P.; CANTARELLA, H.; MORELLI, J.L.; ORLANDO FILHO, J.; LANDELL, M.G.A.; ROSSETO, R. Cana-de-açúcar. In: RAIJ, B. Van; CANTARELLA, H.; QUAGGIO, J.A.; FURLANI, A.M.C. (Ed.). Recomendações de adubação e calagem para o Estado de São Paulo. 2.ed. rev. e atual. Campinas: Instituto Agronômico/Fundação IAC, 1997. p.237-9. (Boletim Técnico, 100).

USEPA. UNITED STATES OF ENVIRONMENTAL PROTECTION AGENCY. Standards for the use and disposal of sewage sludge. Washington, 1986. Code of Federal Regulations 40 CRF Part 503. 